\title{
Passive vibration control of a plate via piezoelectric shunt damping with FEM and ECM
}

P. Lahe Motlagh, Amirreza Aghakhani, Ipek Basdogan

P. Lahe Motlagh, Amirreza Aghakhani, Ipek Basdogan, "Passive vibration control of a plate via piezoelectric shunt damping with FEM and ECM," Proc. SPIE 10601, Smart Materials and Nondestructive Evaluation for Energy Systems IV, 1060103 (27 March 2018); doi: 10.1117/12.2295703 Health Monitoring, 2018, Denver, Colorado, United States 


\title{
Passive vibration control of a plate via piezoelectric shunt damping with FEM and ECM
}

\author{
P. Lahe Motlagh*a Amirreza Aghakhani ${ }^{\mathrm{a}}$, Ipek Basdogan ${ }^{\mathrm{a}}$ \\ ${ }^{a}$ Vibration and Acoustics Laboratory (VAL), Koc University, Istanbul, Turkey
}

\begin{abstract}
Two-dimensional thin plates are widely used in many aerospace, automotive and marine applications. Vibration attenuation can be achieved in these structures by attaching piezoelectric elements on to the structure integrated with shunt damping circuits. This enables a compact vibration damping method without adding significant mass and volumetric occupancy, unlike the bulky mechanical dampers. Practical implementation of shunt damping technique requires accurate modeling of the host structure, the piezoelectric elements and the dynamics of the shunt circuit. Unlike other studies in the literature of piezoelectric shunt damping, this work utilizes a multi-modal equivalent circuit model (ECM) of a thin plate with multiple piezo-patches, to demonstrate the performance of shunt circuits. The equivalent system parameters are obtained from the modal analysis solution based on the Rayleigh-Ritz method. The ECM is coupled to the shunt circuits in SPICE software, where the shunt configuration consists of three branches of electrical resonators, each tuned to one vibration mode of the structure. Using the harmonic analysis in SPICE for a range of excitation frequencies, current output of each ECM branch is calculated for open-circuit and optimum shunt circuit conditions. The current of ECM branches are then converted to the displacement outputs in physical coordinates and validated by the finite-element simulations in ANSYS. It is shown that the vibration attenuation of a vibration mode can be successfully achieved when there is a reduction in the corresponding current amplitude of the ECM branch. This correlation can be utilized in the design of efficient linear/nonlinear shunt circuits.
\end{abstract}

Keywords: Shunt damping, Rayleigh-Ritz, ECM

\section{INTRODUCTION}

Piezoelectric shunt damping techniques have been extensively studied over the past decade as an alternative to the bulky mass-spring-damper systems for attenuating the vibrations of flexible structures. Piezoelectric patches can be directly integrated to plate-like structures, without adding significant mass and volumetric occupancy, and transform the mechanical energy into the electrical energy through various electrical circuit components and as a result, can attenuate the excessive vibrations. These resonant shunt circuits can be easily integrated to plate-like structures and used in many automotive, marine, and aerospace applications to reduce the undesired vibrations.

Accurate modeling tools are required for the circuit elements and the host structure to predict the performance of the shunt damping circuit. Hagwood and von Flotow ${ }^{1}$ showed that a piezoelectric patch shunted to an inductive circuit forms an electrical resonance, acting as equivalent to a pure mechanical vibration damping system. Other research groups have used a network of multi-modal damping resonant shunt circuits ${ }^{2-4}$ for broadband vibration control of flexible structures or implemented negative capacitance circuits ${ }^{5-7}$. Among the studies in the literature, multi-mode shunt damping method ${ }^{8}$ is the most commonly used technique. The method employs a blocking circuit in series with each parallel resistorinductor shunt circuit designed to control one structural mode. The number of the branches in each shunt circuit are decided based on the number of the structural modes to be controlled simultaneously. The blocking circuits avoid the interference of the multi-mode operation such that each structural mode is controlled with one branch.

Equivalent circuit model (ECM) of piezo-patches on a thin plate with electrical circuit elements can be modeled in an electronic circuit simulator (e.g., SPICE) to predict the electrical outputs. The analytical and numerical methods for obtaining the equivalent circuit parameters of beam harvesters have been presented in the literature. ${ }^{9}$ More recently, Aghakhani et al. ${ }^{10}$ developed the multi-modal analytical and numerical approach for identifying the equivalent circuit parameters of multiple piezo-patches attached on a thin plate.

For obtaining the system parameters in the ECM model, an accurate implementation of plate with piezo-patches is required, but few analytical models have been proposed in the literature for vibration analysis of thin plates with surfacebonded piezo-patches ${ }^{9}{ }^{11}$. In those reported works, the electromechanical coupling effect is included in the analytical models, whereas mass and stiffness contribution of the piezo-patches are ignored due to the low volumetric ratio of

Smart Materials and Nondestructive Evaluation for Energy Systems IV, edited by Theodoros E. Matikas, Proc. of SPIE Vol. 10601, 1060103 • @ 2018 SPIE · CCC code: 0277-786X/18/\$18 · doi: 10.1117/12.2295703 
piezo-patches with respect to the isotropic plate. In this study, the parameters of ECM is extracted from a Rayleigh-Ritz model which also includes the mass and stiffness effect of the piezo patches ${ }^{12}$. The ECM model of the thin plate with surface-bonded piezo-patches is used for estimating the shunt damping performance by applying the multimode shunt damping method. The electrical currents at the first three branches corresponding to the first three modes of the structure are measured, and they are compared to open-circuit shunt ECM. Then, the corresponding displacement values for those currents are calculated and compared with the displacement values obtained from FEM simulations. It is observed that reduction in the equivalent current of the ECM branches can accurately represent the damping performance of the shunt circuit.

\section{METHODOGY}

\subsection{Rayleigh-Ritz model of piezo-patches on a thin plate}

In this section, equations of motions for a thin plate with surface-bonded piezo-patches are presented. Figure 1 shows the schematics of the electromechanical system where piezo-patches are connected in parallel to an impedance load:

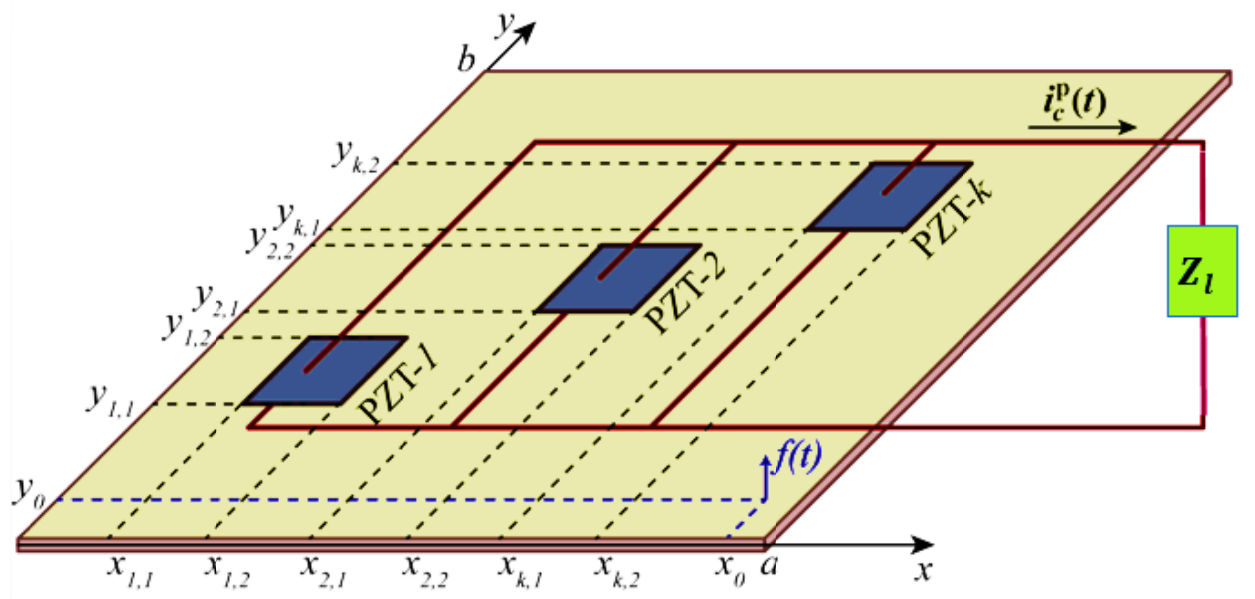

Figure 1. Parallel configuration of multiple piezo-patch energy harvesters attached on a thin plate with a practical interface circuit

Applying Hamilton's principle and using Rayleigh-Ritz method for modal analysis procedure ${ }^{13}$, we obtain the following electromechanical equations for the three structurally integrated piezo-patches connected in parallel to a resistive load in the modal coordinates:

$$
\begin{gathered}
\frac{d^{2} \eta_{m n}(t)}{d^{2} t}+2 \zeta_{m n} \omega_{m n} \frac{d \eta_{m n}(t)}{d t}+\omega_{m n}{ }^{2} \eta_{m n}(t)-v(t) \sum_{k=1}^{p}\left(\theta_{m n}\right)_{k}=f_{m n}(t) \\
\sum_{k=1}^{p} \sum_{n=1}^{n} \sum_{,=1}^{m}\left(\theta_{m n}\right)_{k} \frac{d \eta_{m n}(t)}{d t}+\frac{d v(t)}{d t} \sum_{k=1}^{p}\left(c_{p}\right)_{k}+\frac{V(t)}{Z_{l}(t)}=0
\end{gathered}
$$

Where $\eta_{m n}(t)$ and $v(t)$ are the displacements in modal coordinates and voltage over across the impedance load. Here, $\left(C_{p}\right)_{k}$ is the equivalent capacitance for the $k_{\mathrm{th}}$ patch and given by

$$
\left(C_{p}\right)_{k}=\frac{\left(\varepsilon_{33}{ }^{S}\right)_{k}\left(A_{p}\right)_{k}}{\left(h_{p}\right)_{k}}
$$

where $\left(\varepsilon_{33}{ }^{S}\right)_{k}, A_{p}$, and $h_{p}$ are the dielectric permittivity, the piezo-patch area and thickness of the patch in z-direction, respectively. The modal forcing input and the electromechanical coupling term can be expressed as ; 


$$
f_{m n}=f(t) U_{m n} W_{m n}\left(x_{0}, y_{0}\right)
$$

Here, $\varepsilon_{31}$ is the effective piezoelectric constant, and $U_{m n} W_{m n}\left(x_{0}, y_{0}\right)$ are the assumed modes where $m=1,2, \ldots, M, n$ $=1,2, \ldots, N(M, N$ indicate the number of modes).

\subsection{ECM modeling of the plate and piezo-patches based on Rayleigh-Ritz method}

Figure 2Figure 2. Equivalent circuit representation of the multiple piezo-patch connected to a thin plate for multiple vibration modes (a total of $m \times n$ modes).. Demonstrates the equivalent circuit model of the multiple piezo-patch harvesters connected in parallel to an electrical impedance. Each vibrational mode of the harvester is represented as a second-order circuit connected to each piezo-patch capacitance $\left(C_{p}\right)_{k}$. By applying Kirchhoff's voltage law to the multivibration mode circuit and using equation (1), system parameters of the equivalent circuit model can be determined. Table 1 gives a summary of the equivalent circuit model parameters. ${ }^{9}$

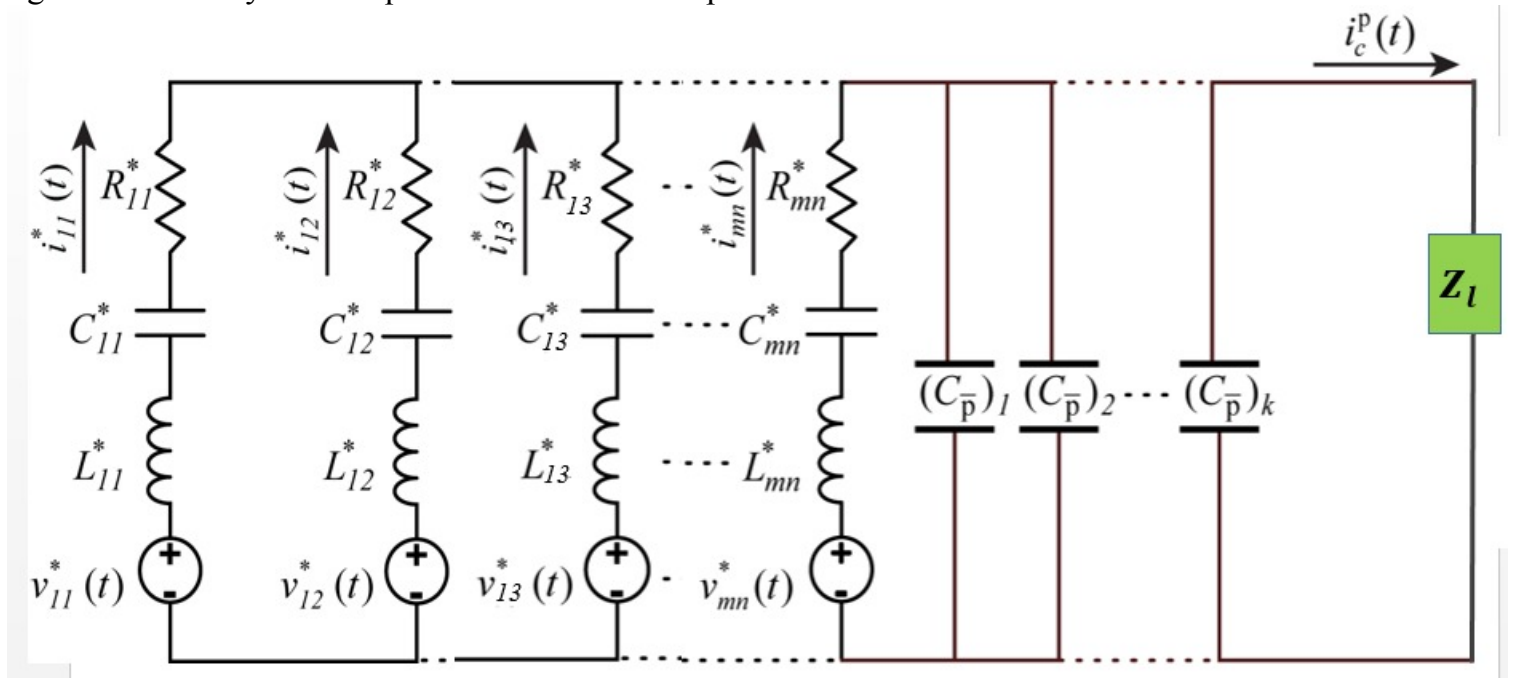

Figure 2. Equivalent circuit representation of the multiple piezo-patch connected to a thin plate for multiple vibration modes (a total of $m \times n$ modes).

Table 1. Analogy between electrical and mechanical domains of multiple piezo-patches integrated to a thin plate

\begin{tabular}{|l|c|}
\hline Equivalent circuit parameters & Mechanical counterparts \\
\hline Voltage source: $V_{m n}(t)$ & $-\left(\sum_{k=1}^{n_{p}}\left(\theta_{m n}\right)_{k}\right)^{-1} f_{m n}(t)$ \\
\hline Electrical current: $i_{m n}(t)$ & $-\sum_{k=1}^{n_{p}}\left(\theta_{m n}\right)_{k} \dot{\eta}^{p}{ }_{m n}(t)$ \\
\hline Inductance: $L_{m n}$ & $\left(\sum_{k=1}^{n_{p}}\left(\theta_{m n}\right)_{k}\right)^{-2}$ \\
\hline Resistance: $R_{m n}$ & $2 \zeta_{m n} \omega_{m n}\left(\sum_{k=1}^{n_{p}}\left(\theta_{m n}\right)_{k}\right)^{-2}$ \\
\hline Capacitance: $C_{m n}$ & $\omega_{m n}{ }^{2}\left(\sum_{k=1}^{n_{p}}\left(\theta_{m n}\right)_{k}\right)^{2}$ \\
\hline
\end{tabular}


where $\theta_{m n}$ is electromechanical coupling factor, $\omega_{m n}$ is natural frequency, and $\zeta_{m n}$ is the damping ratio. If the transverse point force applied on the surface of the plate is of the form $f(t)=F_{0} e^{j \omega t}$, then the steady-state responses in modal coordinates can be written as

$$
\begin{gathered}
\eta_{m n}^{p}(t)=H_{m n}^{p}(t) e^{j\left(\omega t+\tau_{m n}\right)} \\
i^{p}(t)=\sum_{k=1}^{n_{p}} \sum_{n=1}^{N} \sum_{m=1}^{M}\left(i_{m n}^{p}(t)\right)_{k}, \quad\left(i_{m n}^{p}(t)\right)_{k}=-\left(\theta_{m n}\right)_{k} \frac{d \eta_{m n}^{p}(t)}{d t}
\end{gathered}
$$

where $H^{p}{ }_{m n}$ the complex amplitude for the modal is coordinate and $\tau_{m n}$ is the phase shift between the force excitation and the vibration response in modal coordinate. From the current balance equation in (2), the equivalent current of the harvester can be defined as $^{9}(6)$.

\subsection{Multimode shunt damping method}

Multi-mode shunt damping circuit is used as described in ${ }^{8}$. In this model, each mode is represented by one branch. ${ }^{8}$ Figure shows the shunted circuit, each branch consists of 3 sub-branches since the first three modes of the host plate is targeted for this study. RL sub-branch is used to reduce the vibration amplitude of a targeted mode, and LC sub-branches are used to block the effect of the other modes. Values of $C_{i}(i=1,2,3)$ are equal to value of $\mathrm{C}$ (piezoelectric equivalent capacitance). The inductance values $\left(L_{i}\right)$ for the shunt circuits can be calculated from the following expression

$$
L_{i}=\frac{1}{\omega_{i}{ }^{2} C_{i}} i=1,2,3
$$

Where $\omega_{i}$ are the first three natural frequencies of the system. Values of $L_{1,}^{\prime} L_{2}^{\prime}$ and $L_{3}^{\prime}$ are obtained from equations (8) (9Error! Reference source not found.). and (10). ${ }^{8}$ Values of $R_{3}{ }^{\prime}, R_{2}{ }^{\prime}$ and $R_{1}{ }^{\prime}$ are optimized such that they provide maximum reduction in modes 1-3. $R_{1}{ }^{\prime}=20 \mathrm{~K} \Omega, R_{2}{ }^{\prime}=7 \mathrm{~K} \Omega$ for and $R_{3}{ }^{\prime}=6 \mathrm{~K} \Omega$.

$$
\begin{aligned}
& L_{1}^{\prime}=L_{1}-\frac{L_{2}}{\left(1-L_{2} C_{2} \omega_{1}^{2}\right)}-\frac{L_{3}}{\left(1-L_{3} C_{3} \omega_{1}^{2}\right)} \\
& L^{\prime}{ }_{2}=L_{2}-\frac{L_{3}}{\left(1-L_{3} C_{3} \omega_{2}^{2}\right)}-\frac{L_{1}}{\left(1-L_{1} C_{1} \omega_{2}^{2}\right)} \\
& L^{\prime}{ }_{3}=L_{3}-\frac{L_{2}}{\left(1-L_{2} C_{2} \omega_{3}^{2}\right)}-\frac{L_{1}}{\left(1-L_{1} C_{1} \omega_{3}^{2}\right)}
\end{aligned}
$$




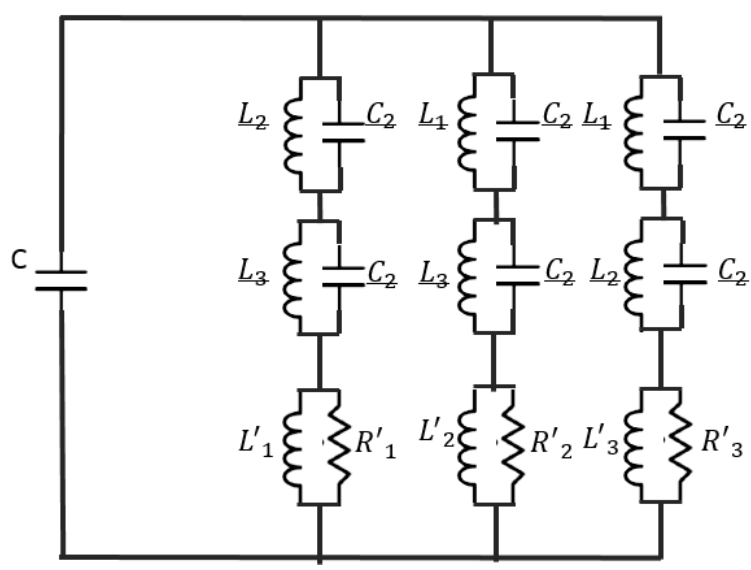

Figure 3. Three mode shunt damping, each branch will be tuned to one mode of the system

\section{RESULTS AND DISCUSSION}

The geometric and material properties of the aluminum plate and piezo-patches are given in Table 2

Table 2. Geometric, material and dielectric properties

\begin{tabular}{|l|c|c|}
\hline Property & Aluminum & Piezoceramic \\
\hline Length $(\mathrm{mm})$ & 580 & 72.4 \\
\hline Width $(\mathrm{mm})$ & 540 & 72.4 \\
\hline Thickness $(\mathrm{mm})$ & 1.96 & 0.267 \\
\hline Young's modulus $(\mathrm{GPa})$ & 70 & 66 \\
\hline Mass density $\left(\mathrm{kg} \mathrm{m}^{-3}\right)$ & 2700 & 7800 \\
\hline Piezoelectric constant $d^{31}\left(\mathrm{pm} \mathrm{V}^{-1}\right)$ & - & -190 \\
\hline Permittivity constant $\bar{\varepsilon}_{33}^{S}\left(\mathrm{nF} \mathrm{m}^{-1}\right)$ & - & 10.38 \\
\hline Damping ratio & 0.005 & 0.005 \\
\hline
\end{tabular}

Figure 4 shows the location of the three patches, the displacement sensor and the excitation force. Vibration attenuation is targeted for the first three modes of the plate and the patch locations are selected accordingly.

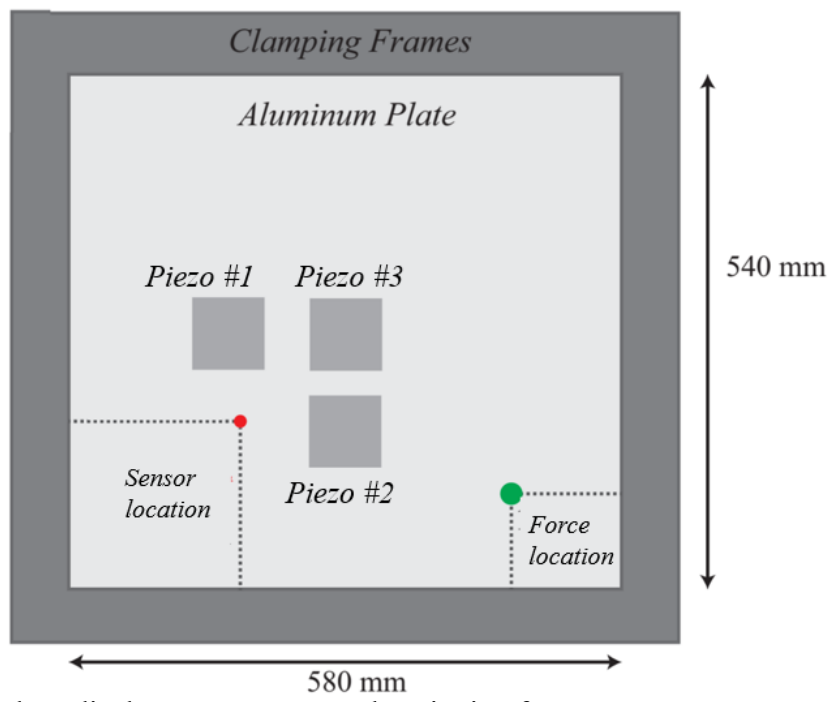

Figure 4 Location of the piezo-patches, displacement sensor and excitation force. 
The first three natural frequencies of the host plate are 55.2, 108.2 and $118 \mathrm{~Hz}$, and the shunt damping circuit parameters are calculated using Equations (8), (9) and (10) and they are listed in Table 3. C for the piezoelectric patch is taken as $595.5 \mathrm{nF}$.

Table 1. Parameters of the multimodal shunt circuit

\begin{tabular}{|c|l|l|l|l|}
\hline$\omega_{1}=55.2 \mathrm{~Hz}$ & $\mathrm{~L}_{1}=13.94 \mathrm{H}$ & $L_{1}^{\prime}=5.14 \mathrm{H}$ & $\mathrm{C}_{1}=595 . \mathrm{nF}$ & $\mathrm{R}_{1}=20 \mathrm{~K} \Omega$ \\
\hline$\omega_{2}=108.2 \mathrm{~Hz}$ & $\mathrm{~L}_{2}=3.63 \mathrm{H}$ & $L_{2}^{\prime}{ }_{2}=10.2 \mathrm{H}$ & $\mathrm{C}_{1}=595 . \mathrm{nF}$ & $\mathrm{R}_{2}=7 \mathrm{~K} \Omega$ \\
\hline$\omega_{3}=118 \mathrm{~Hz}$ & $\mathrm{~L}_{3}=\underline{\underline{ }} \underline{\underline{3} .06 \mathrm{H}}$ & $L_{3}^{\prime}=26.1 \mathrm{H}$ & $\mathrm{C}_{1}=595 . \mathrm{nF}$ & $\mathrm{R}_{3}=\underline{\underline{\mathrm{K}} \Omega}$ \\
\hline
\end{tabular}

After generating the ECM model and the shunt damping circuits, electrical current of each branch is measured. Once the current flowing through each branch (Please refer to Figure 2 for the measured currents) is measured in SPICE software, first derivative of the modal displacement $\left(\eta_{m n}\right)$ can be calculated using the relationship in Table 2. Displacements in physical coordinates corresponding to that measured current values can be calculated using the force-displacement relationship in Equation 6. Then the following equation ${ }^{11}$ is used to calculate the displacements on the plate

$$
w(x, y, t)=\sum_{n=1}^{N} \sum_{m=1}^{M} \phi_{m n}(x, y) \eta_{m n}(t)
$$

Figure 5 shows the proportional current measured at the three branches of the ECM model (Please refer to Figure 2 for the ECM model) as a function of excitation frequency. The shunt circuit current values are compared with open circuit (eg. when impedance load is very large, $\mathrm{R}($ resistor $)=10^{8} \Omega$ ) results. As it can be observed from Figure 55, proportional current in each branch is reduced as a result of the shunt circuit integrated to the system.

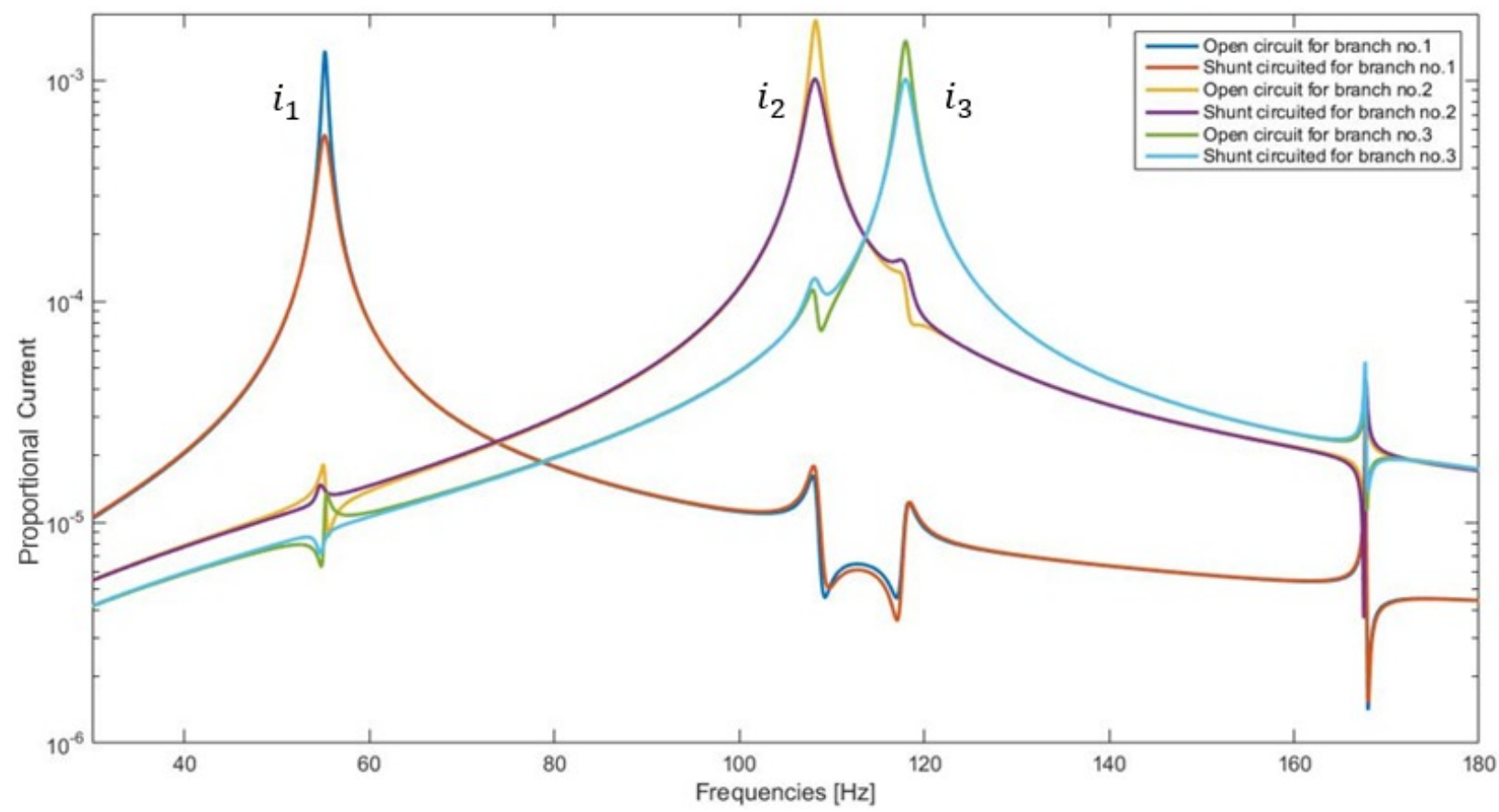

Figure 5. Proportional current from first three branches of ECM (presented in figure 2)

By following the above procedure using Equation 10, displacement FRFs are obtained as shown in Figure 6a. The vibration amplitudes in the first three modes are reduced significantly. In addition, a FE model of the plate with the integrated piezo patches are created in ANSYS software to validate the calculated displacement FRFs from the ECM. The Figure 6b shows the displacement FRFs calculated using the ANSYS model. When Figure 6a and 6b are compared, it can be seen that the ECM method accurately predicts the displacement values for the shunt damping circuit case. Table 
4 lists the reduction percentages for the ECM and FEM models. From Figure 6 and Table 4, it can be observed that, ECM method, agrees well with the ANSYS simulation results. In the first three modes of the structure the vibration amplitudes are reduced up to $58 \%$.
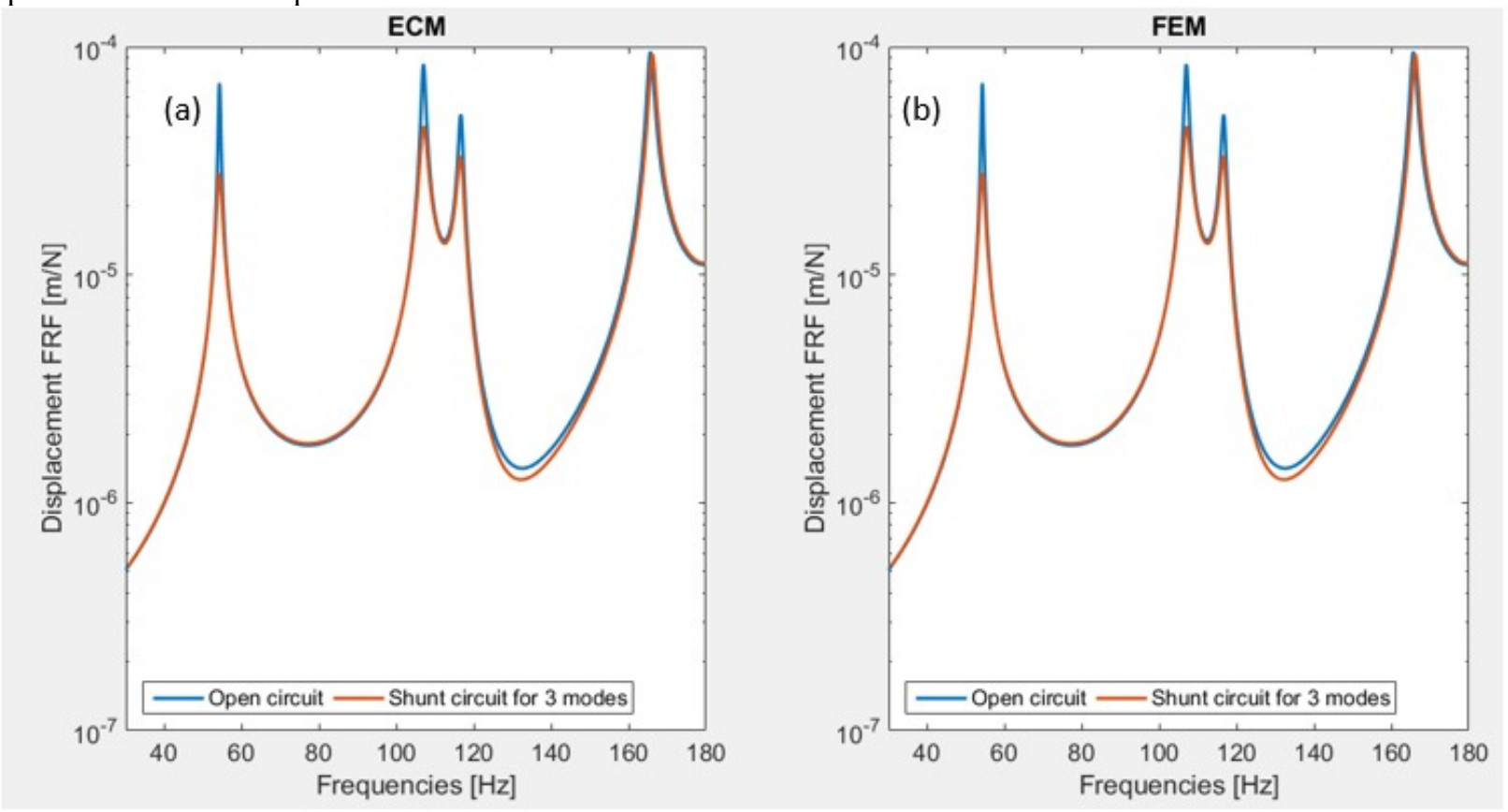

Figure 6- Displacement FRF extracted from ECM (a) and FEM (b)

Table 4.Percentage reduction for first three modes of the structure

\begin{tabular}{|c|c|c|}
\hline \multirow{2}{*}{ Mode number } & \multicolumn{2}{|c|}{ Percentage reduction } \\
\cline { 2 - 3 } & Analytical simulation & FEM result \\
\hline 1 & 58.01 & 58.00 \\
\hline 2 & 47.03 & 47.02 \\
\hline 3 & 34.02 & 34.01 \\
\hline
\end{tabular}

\section{CONCLUSION}

We implemented a multi-modal equivalent circuit model (ECM) of a thin plate with multiple piezo-patches, to demonstrate the performance of shunt circuits. The equivalent system parameters are obtained from the modal analysis solution based on the Rayleigh-Ritz method. The ECM is integrated to the shunt circuits in SPICE software, where the each shunt branches are tuned to one vibration mode of the structure. Using the harmonic analysis in SPICE, current output of each ECM branch is calculated for open-circuit and optimum shunt circuit conditions. The current of ECM branches are then converted to the displacement outputs in physical coordinates and validated by the finite-element simulations in ANSYS. It is shown that vibration amplitudes are successfully reduced when there is a reduction in the corresponding current amplitude of the ECM branch. This correlation can be utilized in the design of efficient linear/nonlinear shunt circuits. 


\section{REFERENCES}

[1] Nesbitt W. Hagood, Walter H. Chung and Andreas Von Flotow., "Modelling of Piezoelectric Actuator Dynamics for Active Structural Control," J. Intell. Mater. Syst. Struct. 1(3), 327-354 (1990).

[2] Hollkamp, J. J., "Multimodal Passive Vibration Suppression with Piezoelectric Materials and Resonant Shunts," J. Intell. Mater. Syst. Struct. 5(1), 49-57 (1994).

[3] Wu, S., "Method for Multiple Mode Shunt Damping of Structural Vibration Using a Single PZT Transducer," SPIE 3327(1), L. P. Davis, Ed., 159-168 (1998).

[4] Wu, S., "Multiple PZT transducers implemented with multiple-mode piezoelectric shunting for passive vibration damping," 2 June 1999, 112-122, International Society for Optics and Photonics.

[5] Behrens, S., Fleming, A. J. and Moheimani, S. O. R., "A broadband controller for shunt piezoelectric damping of structural vibration," Smart Mater. Struct. 12(1), 18-28 (2003).

[6] Collet, M., Ouisse, M., Ichchou, M. N., Ohayon, R. and Ohayon, M. C. and M. O. and M. N. I. and R., "Semiactive optimization of 2D wave dispersion into shunted piezo-composite systems for controlling acoustic interaction," Smart Mater. Struct. 21(9), 094002 (2012).

[7] Beck, B. S., Cunefare, K. A. and Collet, M., "The power output and efficiency of a negative capacitance shunt for vibration control of a flexural system," Smart Mater. Struct. 065009(22), 10 (2013).

[8] Shu-Yau Wu., "Method for Multiple Mode Piezoelectric Shunting with Single PZT Transducer for Vibration Control," J. Intell. Mater. Syst. Struct. 9(12), 991-998 (1998).

[9] Bayik, B., Aghakhani, A., Basdogan, I. and Erturk, A., "Equivalent circuit modeling of a piezo-patch energy harvester on a thin plate with AC-DC conversion," Smart Mater. Struct. 25(5), 055015 (2016).

[10] Aghakhani, A., Basdogan, I. and Erturk, A., "Multiple piezo-patch energy harvesters integrated to a thin plate with AC-DC conversion: analytical modeling and numerical validation," 1 April 2016, 98060C.

[11] Ugur Aridogan, Ipek Basdogan and Alper Erturk., "Multiple patch-based broadband piezoelectric energy harvesting on plate-based structures," J. Intell. Mater. Syst. Struct. 25(14), 1664-1680 (2014).

[12] gozum, m, Aghakhani, a, serhat, g and Basdogan, i., "Electroelastic Modeling of Thin Laminated Composite Plates with Surface-bonded Piezo-Patches using Rayleigh-Ritz Method,” J. Intell. Mater. Syst. Struct. in press.

[13] Yoon, H., Youn, B. D. and Kim, H. S., "Kirchhoff plate theory-based electromechanically-coupled analytical model considering inertia and stiffness effects of a surface-bonded piezoelectric patch," Smart Mater. Struct. 25(2), 025017 (2016). 